\title{
Análisis de las dependencias composicionales de las propiedades opticas de láminas semiconductoras amorfas del sistema As-S-Se
}

\author{
J.M. GONZÁLEZ-LEAL', R. PRIETO-ALCÓN², J.A. ÁNGEL², R. JIMÉNEZ-GARAY², E. MÁRQUEZ² \\ ${ }^{1}$ Department of Chemistry. University of Cambridge. Lensfield Road. CB2 1EW. Cambridge. UK. \\ ${ }^{2}$ Departamento de Física de la Materia Condensada. Facultad de Ciencias. Universidad de Cádiz. 11.510 - Puerto Real (Cádiz).
}

\begin{abstract}
Se han preparado láminas semiconductoras amorfas de composiciones químicas $\mathrm{As}_{40} \mathrm{~S}_{60-\mathrm{x}} \mathrm{Se}_{\mathrm{x}}$ (con $\mathrm{x}=0,20,30,40$ y $60 \%$ at.), mediante la técnica de evaporación térmica en vacío. Los valores del índice de refracción y del coeficiente de absorción de estas láminas, se determinaron a partir de los espectros de transmisión óptica, obtenidos en incidencia normal, en el intervalo espectral comprendido entre 400 y $2200 \mathrm{~nm}$. Se ha encontrado un aumento en los valores del índice de refracción con el contenido de Se, dentro del intervalo espectral en estudio. El análisis de la dependencia composicional de esta constante óptica, haciendo uso de la relación de Lorentz-Lorenz, sugiere que este aumento se debe a la mayor polarizabilidad de los átomos de Se, relacionada con su mayor tamaño, en comparación con los átomos de S. Los valores del número de coordinación efectivo del As, se han estimado a partir del análisis de la dispersión del índice de refracción, sobre la base de un modelo basado en la fórmula del oscilador unidimensional. Los resultados revelan un aumento de este parámetro estructural con el contenido de Se, desde aproximadamente 3.0 hasta 3.4, lo que sugiere que la sustitución de S por Se favorece la compactación de la estructura. Estos resultados son consistentes con las medidas de difracción de rayos $\mathrm{X}$ realizadas sobre las muestras, en las que se observa una disminución en la intensidad del pre-pico de difracción. Finalmente, se ha encontrado que el gap óptico disminuye con el contenido de Se, desde 2.38 hasta $1.79 \mathrm{eV}$, lo que puede explicarse atendiendo a la menor energía de los enlaces As-Se, comparada con la de los enlaces As-S.
\end{abstract}

Palabras clave: Semiconductores amorfos, vidrios calcogenuros, propiedades ópticas.

\section{Analysis of the Compositional Dependencies of the Optical Properties of Amorphous Semiconducting Films of the System As-S-Se}

Amorphous $\mathrm{As}_{40} \mathrm{~S}_{60-\mathrm{x}} \mathrm{Se}_{\mathrm{x}}(\mathrm{x}=0,20,30,40$ and 60 at. \%) films have been prepared by vacuum thermal evaporation. Values for the refractive index and the absorption coefficient of these films have been determined from their transmission spectra, measured at normal incidence, in the spectral range from 400 to $2200 \mathrm{~nm}$. It has been found that the refractive index of the samples increases with increasing Se content, over the entire spectral range. This behaviour can be explained, according to the Lorentz-Lorenz relationship, by the increased polarizability of the larger Se atoms, in comparison with $S$ atoms. The values of the As effective coordination number, $N_{\mathrm{c}}$, have been estimated from the analysis of the dispersion of the refractive index, in terms of a model based on the single-oscillator formula. The increase in $N_{\mathrm{c}}$ with increasing Se content, from around 3.0 to 3.4, which has been inferred from this analysis, suggests an increase in the structural compactness of the films when substituting $\mathrm{S}$ by Se. This conclusion is supported by the decrease observed in the intensity of the first sharp diffraction peak, in the X-ray patterns. Finally, it has been found a decrease in the optical bandgap with increasing Se content, from 2.38 to $1.79 \mathrm{eV}$, which can be plausibly explained considering the lower bonding energy of As-Se bonds compared with that of As-S bonds.

Keywords: Amorphous semiconductors, chalcogenide glasses, optical properties.

\section{INTRODUCCIÓN}

Las propiedades estructurales, eléctricas y ópticas de los semiconductores amorfos calcogenuros han sido objeto de interés durante las últimas décadas [1-3]. Este interés ha estado estimulado, por una parte, por el conocimiento general de los sólidos no cristalinos, que de ellos se puede extraer, y por otra parte, por sus posibles aplicaciones tecnológicas [3].

En este artículo se analizarán las propiedades ópticas de láminas amorfas de las aleaciones $\mathrm{As}_{40} \mathrm{~S}_{60-\mathrm{x}} \mathrm{Se}_{\mathrm{x}}$ (con $\mathrm{x}=0,20,30,40$ y $60 \%$ at.), preparadas mediante evaporación térmica. Asimismo, se discutirá la relación entre las propiedades ópticas y la estructura de estos materiales, en base a los resultados obtenidos a través de experimentos de difracción de rayos X. Es importante notar, que aunque es posible encontrar en la literatura algunos artículos que tratan tanto de láminas delgadas [4-6], como de materiales en forma masiva [7-11] dentro de esta línea de composiciones, no existe, hasta donde llega el conocimiento de los autores, un estudio detallado de sus propiedades ópticas. Por tanto, la ausencia hasta ahora de los resultados de este estudio, unida al potencial tecnológico de estos materiales $[9,10]$, creemos que resaltan la investigación que se presenta en este artículo.

\section{EXPERIMENTAL}

Los materiales de partida, con composiciones $\mathrm{As}_{40} \mathrm{~S}_{60-\mathrm{x}} \mathrm{Se}_{\mathrm{x}}(\mathrm{x}=0,20$, 30,40 y $60 \%$ at.), se prepararon en forma masiva mediante el método convencional del enfriamiento rápido de la mezcla fundida, a partir de elementos de pureza $5 \mathrm{~N}$. El proceso de síntesis se llevó a cabo en ampollas de cuarzo y en vacío, usando un horno cuya temperatura se mantuvo entre 700 y $750{ }^{\circ} \mathrm{C}$ durante un intervalo de 8 a $24 \mathrm{~h}$, dependiendo de la composición. Las láminas delgadas de estos materiales se depositaron sobre substratos vítreos (portaobjetos de microscopio), mediante la evaporación térmica en vacío del material masivo obtenido anteriormente. El proceso de deposición se llevó a cabo empleando un sistema de evaporación (Tesla Corporation, modelo UP-858) a una presión de aproximadamente $10^{-3} \mathrm{~Pa}$. Durante la deposición los substratos se mantuvieron girando mediante un sistema denominado de rotación planetaria, el cual hace posible reducir la falta de uniformidad en el espesor de las láminas. Los espesores se fijaron típicamente en $\approx 1 \mu \mathrm{m}$. La velocidad de deposición se midió a lo largo del proceso usando una microbalanza de cuarzo, y se mantuvo en el intervalo comprendido entre 1 y $8 \mathrm{~nm} \mathrm{~s}^{-1}$. Esta velocidad de deposición dió lugar a láminas cuya composición química es muy próxima a la del 
material de partida. Así, las composiciones químicas de las láminas calcogenuras preparadas fueron $\mathrm{As}_{38.7 \pm 0.8} \mathrm{~S}_{61.6 \pm 0.7^{\prime}} \mathrm{As}_{39.8 \pm 0.5} \mathrm{~S}_{39.9 \pm 0.6} \mathrm{Se}_{20.3 \pm 0.3^{\prime}}$ $\mathrm{As}_{38.9 \pm 1.14} \mathrm{~S}_{31.0 \pm 0.5} \mathrm{Se}_{30.2+1.7^{\prime}} \mathrm{As}_{39.111 .9} \mathrm{~S}_{22.2 \pm 0.9} \mathrm{Se}_{38.711 .9}$ y $\mathrm{As}_{41.2+1.8} \mathrm{Se}_{58.811 .8^{\prime}}$ como se pudo comprobar mediante microanálisis por dispersión de energías, usando para ello un microscopio electrónico de barrido (Jeol, model JSM-820). El carácter amorfo de las láminas se comprobó de forma sistemática realizando experimentos de difracción de rayos $\mathrm{X}$ (Philips, model PW-1820). Es importante destacar que las láminas se mantuvieron en completa oscuridad hasta el momento de las medidas, con objeto de minimizar la exposición de las mismas a fuentes de luz, lo que podría dar lugar a cambios en las propiedades ópticas y producir, además, la posible oxidación de éstas, estimulada por la luz [12].

Los espectros de transmisión óptica se obtuvieron, en incidencia normal, mediante un espectrofotómetro UV/Vis/NIR (Perkin-Elmer, model Lambda-19), y el intervalo de longitudes de onda analizado estuvo comprendido entre 400.y 2200.nm. Los espesores de las láminas se midieron de forma independiente usando un perfilómetro (Sloan, modelo Dektak 3030). Estos valores medidos mecánicamente se compararon con aquellos calculados a partir de los espectros de transmisión óptica. Todas las medidas se realizaron a temperatura ambiente.

\section{RESULTADOS}

Las láminas amorfas del sistema $\mathrm{As}_{40} \mathrm{~S}_{60-x} \mathrm{Se}_{x^{\prime}}$ preparadas por evaporación térmica, se caracterizaron geométrica y ópticamente a partir de sus espectros de transmisión óptica, $T(\lambda)$, obtenidos en incidencia normal. Estos espectros aparecen representados en el recuadro de la Fig. 1 y muestran un claro desplazamiento hacia el rojo de la región libre de interferencias, al aumentar el contenido de Se. El espesor medio de las láminas, $\bar{d}$, así como las magnitudes ópticas, índice de refracción, $n$, y coeficiente de absorción, $\alpha$, se han determinado haciendo uso de un método de caracterización basado en las envolventes superior e inferior, $T_{+}$y $T_{-}$, respectivamente, de $T(\lambda)$ [13] (ver Fig. 1). Las ecuaciones empleadas tienen en consideración la absorción del substrato sobre el que las láminas están depositadas, lo que se ha demostrado que mejora significativamente la exactitud en el cálculo de $\bar{d}$ y $n$, y reduce el error relativo de estas magnitudes por debajo del $1 \%$. Por otro lado, dado que las láminas preparadas mediante evaporación térmica presentan generalmente un espesor variable (aún cuando esta variación puede reducirse empleando el sistema de rotación planetaria comentado anteriormente), la caracterización geométrica de las láminas supuso también la estimación de la variación en el espesor, $\Delta d$, en los extremos del área iluminada por el espectrofotómetro $\left(1 \times 4 \mathrm{~mm}^{2}\right)$. Todos los detalles relativos al método de caracterización empleado pueden consultarse en la Ref. [13].

Para ilustrar el procedimiento para el cálculo de $\bar{d}, \Delta d$ y $n$, los resultados correspondientes a una lámina amorfa representativa de composición $\mathrm{As}_{40} \mathrm{~S}_{40} \mathrm{Se}_{20}$ aparecen recogidos en la Tabla 1. El espectro de transmisión óptica correspondiente a esta muestra representativa se presenta en la Fig. 1, junto con sus envolventes $T_{+}$y $T_{\text {, las cuales }}$ han sido trazadas usando un algoritmo desarrollado por McClain et al. [14]. Además, los espectros de transmisión, $T_{s}(\lambda)$, y de reflexión, $R_{\mathrm{s}}(\lambda)$, del substrato se representan también en esta figura. Los efectos producidos por la absorción del substrato se evidencian claramente en $T_{\mathrm{s}}(\lambda)$. Como se comentó anteriormente, los espesores de las láminas se midieron mecánicamente mediante un perfilómetro. El valor del espesor medio medido para la lámina representativa indicada fue de $1166 \pm 18(1.5 \%) \mathrm{nm}$, el cual presenta un acuerdo excelente con el correspondiente valor calculado, $1148 \pm 5(0.4 \%) \mathrm{nm}$. En todos los casos, las diferencias entre las medidas mecánicas y los valores calculados fueron menores del $2 \%$.

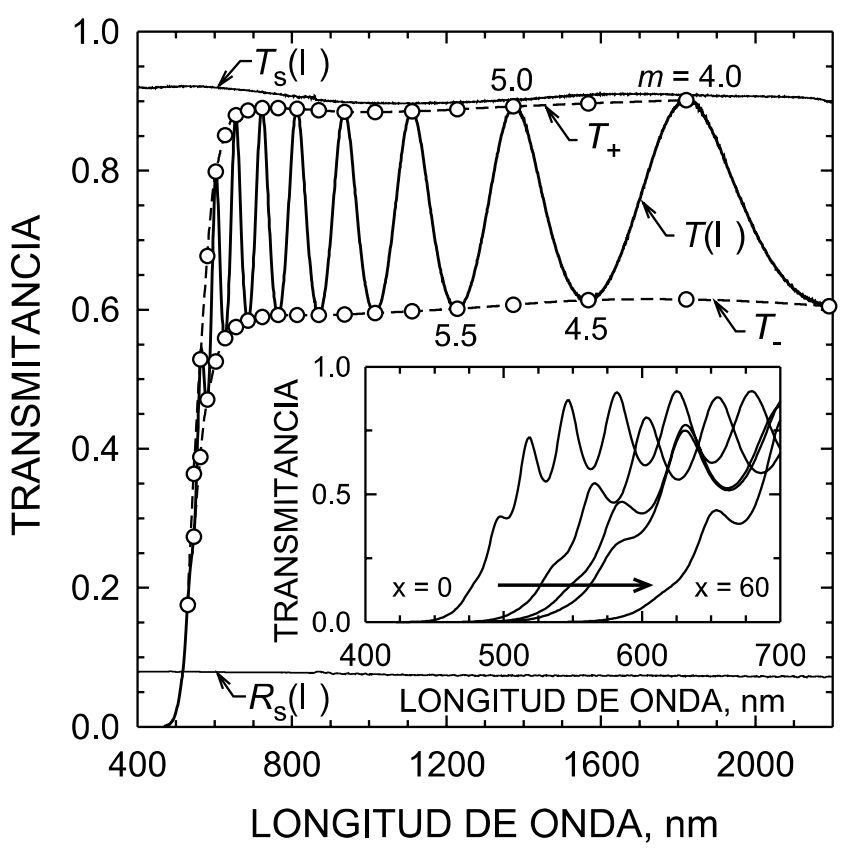

Figura 1. Espectro de transmisión óptica, $T(\lambda)$, en incidencia normal, para una lámina amorfa virgen de composición $\mathrm{As}_{40} \mathrm{~S}_{40} \mathrm{Se}_{20}$. Las envolventes superior, $T_{+}$, e inferior, $T$, de $T(\lambda)$, y los espectros de transmisión, $T_{\mathrm{s}}(\lambda)$, y de reflexión, $R_{\mathrm{s}}(\lambda)$, correspondientes al substrato en solitario, también aparecen representados. Se han marcado los puntos de tangencia entre las envolventes y el espectro, los cuales están relacionados con el método de caracterización óptica empleado, así como los números de orden, $m$, correspondientes algunos de ellos. Los espectros de transmisión óptica a longitudes de onda cortas, de las láminas amorfas del sistema $\mathrm{As}_{40} \mathrm{~S}_{60-\mathrm{x}} \mathrm{Se}_{\mathrm{x}^{\prime}}$, se muestran en el recuadro. En él se observa un desplazamiento hacia el rojo de la región libre de interferencias al aumentar el contenido de Se.

TABLA 1. CÁlCUlo Del ESPESOR MEDio, $\bar{d}$, VARIACión DEL ESPESOR, $\Delta d$, E ÍNDICE DE REFRACCIÓN, $n$, PARA UNA LÁMINA REPRESENTATIVA DE LA ALEACIÓN AMORFA $\mathrm{As}_{40} \mathrm{~S}_{40} \mathrm{Se}_{20}$, SIGUIENDO EL PROCEDIMIENTO DESCRITO EN LA REF. [13]. El RESTO DE LAS MAGNITUDES QUE APARECEN EN LA TABLA SON: LOS VALORES DE LA LONGITUD DE ONDA EN LAS QUE LAS ENVOLVENTES $T$ y $T$ SON TANGENTES AL ESPECTRO $T(\lambda), \lambda$, LOS VALORES DEL ÍNDICE DE REFRACCIÓN Y DE LA ABSORBANCIA DEL SUBSTRATO, $s$ y $a$, RESPECTIVAMENTE, Y LOS NÚMEROS DE ORDEN CORRESPONDIENTES A LOS PUNTOS DE TANGENCIA, $m$. A PARTIR DE LOS VALORES PRESENTADOS EN LA TABLA SE HA OBTENIDO UN VALOR PARA EL ESPESOR MEDIO DE LA LÁMINA $\bar{d}=1148 \pm 5 \mathrm{~nm}$, Y UNA VARIACIÓN EN SU ESPESOR $\Delta d=13 \pm 5$ $\mathrm{nm}$. EL VALOR CORRESPONDIENTE A LA VARIACIÓN EN EL ESPESOR SE HA DETERMINADO PROMEDIANDO LOS VALORES DE $\Delta d$ SUBRAYADOS.

\begin{tabular}{|c|c|c|c|c|c|c|c|c|}
\hline $\begin{array}{c}\lambda_{\text {tan }} \\
(\mathrm{nm})\end{array}$ & $s$ & $a_{\mathrm{s}}$ & $T_{+}$ & $T_{-}$ & $\begin{array}{c}\Delta d \\
(\mathrm{~nm})\end{array}$ & $m$ & $\begin{array}{c}\bar{d} \\
(\mathrm{~nm})\end{array}$ & $n$ \\
\hline 1588 & 1.491 & 0.9831 & 0.903 & 0.623 & 22.3 & 3.5 & 1155 & 2.421 \\
\hline 1395 & 1.495 & 0.9767 & 0.899 & 0.616 & $\underline{14.5}$ & 4.0 & 1150 & 2.430 \\
\hline 1243 & 1.500 & 0.9737 & 0.896 & 0.611 & 12.4 & 4.5 & 1145 & 2.436 \\
\hline 1124 & 1.498 & 0.9710 & 0.893 & 0.607 & 11.3 & 5.0 & 1147 & 2.448 \\
\hline 1027 & 1.503 & 0.9726 & 0.892 & 0.605 & 12.2 & 5.5 & 1145 & 2.460 \\
\hline 947 & 1.508 & 0.9754 & 0.893 & 0.604 & 12.4 & 6.0 & 1145 & 2.475 \\
\hline 880 & 1.514 & 0.9799 & 0.895 & 0.603 & 11.5 & 6.5 & 1143 & 2.491 \\
\hline 822 & 1.510 & 0.9842 & 0.898 & 0.604 & 12.6 & 7.0 & 1147 & 2.506 \\
\hline 773 & 1.509 & 0.9875 & 0.900 & 0.604 & 12.4 & 7.5 & 1151 & 2.525 \\
\hline 730 & 1.509 & 0.9909 & 0.900 & 0.602 & 13.0 & 8.0 & 1152 & 2.544 \\
\hline 693 & 1.508 & 0.9932 & 0.898 & 0.598 & 14.2 & 8.5 & 1153 & 2.566 \\
\hline 661 & 1.508 & 0.9951 & 0.892 & 0.589 & 15.6 & 9.0 & 1151 & 2.591 \\
\hline 633 & 1.508 & 0.9968 & 0.868 & 0.575 & 20.2 & 9.5 & 1153 & 2.619 \\
\hline 608 & 1.508 & 0.9980 & 0.821 & 0.544 & 24.2 & 10.0 & 1143 & 2.648 \\
\hline 586 & 1.509 & 0.9992 & 0.716 & 0.496 & 28.0 & 10.5 & 1153 & 2.680 \\
\hline 568 & 1.509 & 1.0000 & 0.584 & 0.420 & 26.3 & 11.0 & 1136 & 2.721 \\
\hline 549 & 1.510 & 1.0000 & 0.405 & 0.305 & 20.0 & 11.5 & - & 2.750 \\
\hline
\end{tabular}


La dispersión del índice de refracción se ha analizado sobre la base del modelo de Wemple y DiDomenico (WDD) [15,16], el cuál está basado en la fórmula del oscilador unidimensional:

$$
n^{2}(\eta \omega)=1+\frac{E_{\mathrm{o}} E_{\mathrm{d}}}{E_{\mathrm{o}}^{2}-(\mathrm{h} \omega)^{2}}
$$

donde $E_{\mathrm{o}}$ es la energía del oscilador y $E_{\mathrm{d}}$ es la energía de dispersión. El parámetro $E_{\mathrm{o}}$ se considera como un gap de energía "promedio" y varía proporcionalmente al gap de Tauc, $\mathrm{E}_{\mathrm{g}}^{\mathrm{opt}}$ (el cual será definido formalmente más adelante, cuando se estudie el borde de absorción óptica): $E_{\mathrm{o}} \approx 2 \times \mathrm{E}_{\mathrm{g}}^{\text {opt }}$ [17]. Por otro lado, uno de los logros del modelo de WDD es que relaciona la energía de dispersión, $E_{\mathrm{d}^{\prime}}$ con otros parámetros físicos del material a través de la siguiente expresión empírica [15,16]:

$$
E_{\mathrm{d}}=\beta N_{\mathrm{c}} \mathrm{Z}_{\mathrm{a}} N_{\mathrm{e}}(\mathrm{eV})
$$

donde $N_{c}$ es el número de coordinación efectivo del catión más cercano al anión, $Z_{\mathrm{a}}$ es la valencia química del anión, $N_{\mathrm{e}}$ es el número efectivo de electrones de valencia por anión, y $\beta$ es una constante que toma el valor $\beta_{\mathrm{i}}=0.26 \pm 0.03 \mathrm{eV}$ para materiales predominantemente iónicos, $\mathrm{y}$ $\beta_{c}=0.37 \pm 0.04 \mathrm{eV}$ para materiales predominantemente covalentes.

Representando gráficamente los valores del índice de refracción obtenidos en la forma $\left(n^{2}-1\right)^{-1} \mathrm{vs}(\eta \omega)^{2}$, y realizando un ajuste lineal por mínimos cuadrados, como se muestra en la Fig. 2, los valores de los parámetros $E_{\mathrm{o}}$ y $E_{\mathrm{d}}$ se pueden determinar directamente a partir de la pendiente, $\left(E_{\mathrm{o}} E_{\mathrm{d}}\right)^{-1}$, y de la ordenada en el origen, $E_{\mathrm{o}} / E_{\mathrm{d}}$. No obstante, debe tenerse en cuenta que el modelo de WDD sólo es válido en la región de transparencia, donde el coeficiente de absorción de la lámina calcogenura toma valores $\alpha \approx 0$. Por esta razón, la variación experimental del índice de refracción con la energía se aparta del comportamiento dado por la Ec. (1), cuando la energía del fotón se aproxima a $\mathrm{E}_{\mathrm{g}}^{\mathrm{opt}}$, como se observa en la Fig. 2. Así, sólo los valores del índice de refracción a bajas energías son considerados en el ajuste lineal por mínimos cuadrados. Los valores de los parámetros $E_{\mathrm{o}}$ y $E_{\mathrm{d}^{\prime}}$ así como el valor del índice de refracción cuando $\eta \omega \rightarrow 0$ (obtenido extrapolando la Ec. (1) hacia la región espectral del infrarrojo), $n(0)$, para las láminas amorfas del sistema $\mathrm{As}_{40} \mathrm{~S}_{60-\mathrm{x}} \mathrm{Se}_{\mathrm{x}^{\prime}}$ se presentan en la Tabla 2. Las dependencias de $E_{\mathrm{o}}$ y $E_{\mathrm{d}}$ con la composición, se encuentran ambas representadas en la Fig. 3.

TABla 2. VALORES de LOS PARÁMETROS De disPersión ÓPtica, $E_{\mathrm{o}}$ y $E_{\mathrm{d}^{\prime}}$ ÍNDICE DE REFRACCIÓN “ESTÁTICO”, $n(0)=n(\eta \omega \Theta 0)$, NÚMERO DE COORDINACIÓN EFECTIVO DEL ÁTOMO DE As, $N_{c^{\prime}}$ y GAP DE TAUC, E opt, PARA LAS LÁMINAS AMORFAS VÍRGENES DEL SISTEMA $\mathrm{As}_{40} \mathrm{~S}_{60-x} \mathrm{Se}_{x^{\prime}}$, PREPARADAS MEDIANTE EVAPORACIÓN TÉRMICA EN VACÍO.

\begin{tabular}{|c|c|c|c|c|c|}
\hline $\begin{array}{c}\text { Contenido } \\
\mathrm{de} \text { Se }(\% \text { at. })\end{array}$ & $E_{\mathrm{o}}(\mathrm{eV})$ & $E_{\mathrm{d}}(\mathrm{eV})$ & $n(0)$ & $N_{\mathrm{c}}$ & $\mathrm{E}_{\mathrm{g}}^{\text {opt }}(\mathrm{eV})$ \\
\hline $\mathrm{x}=0$ & $4.94 \pm 0.03$ & $20.67 \pm 0.12$ & $2.277 \pm 0.001$ & $3.0 \pm 0.3$ & $2.38 \pm 0.01$ \\
\hline $\mathrm{x}=20$ & $4.53 \pm 0.03$ & $21.64 \pm 0.14$ & $2.404 \pm 0.001$ & $3.1 \pm 0.3$ & $2.13 \pm 0.01$ \\
\hline $\mathrm{x}=30$ & $4.31 \pm 0.04$ & $21.65 \pm 0.20$ & $2.454 \pm 0.001$ & $3.1 \pm 0.3$ & $2.01 \pm 0.01$ \\
\hline $\mathrm{x}=40$ & $4.18 \pm 0.02$ & $22.54 \pm 0.13$ & $2.528 \pm 0.001$ & $3.2 \pm 0.3$ & $1.93 \pm 0.01$ \\
\hline $\mathrm{x}=60$ & $3.86 \pm 0.02$ & $23.74 \pm 0.11$ & $2.674 \pm 0.001$ & $3.4 \pm 0.3$ & $1.79 \pm 0.01$ \\
\hline
\end{tabular}

Los espectros de absorción óptica, $\alpha(\eta \omega)$, calculados siguiendo el procedimiento descrito en [13], para las láminas vírgenes de las composiciones químicas que son objetos de estudio, se muestran en la Fig. 4 , haciendo uso de una escala semilogarítmica. Se puede observar un claro desplazamiento hacia el rojo del borde de absorción al aumentar el contenido de Se. El análisis de la región de fuerte absorción, donde $\alpha>\approx 10^{4} \mathrm{~cm}^{-1}$, se ha llevado a cabo sobre la base de la denominada ley de Tauc [18]:

$$
\alpha(\eta \omega)=B \frac{\left(\eta \omega-E_{\mathrm{g}}^{\mathrm{opt}}\right)^{2}}{\eta \omega}
$$

donde $B$ es una constante que depende de la probabilidad de transición electrónica, y E opt es el anteriormente mencionado gap de Tauc, que

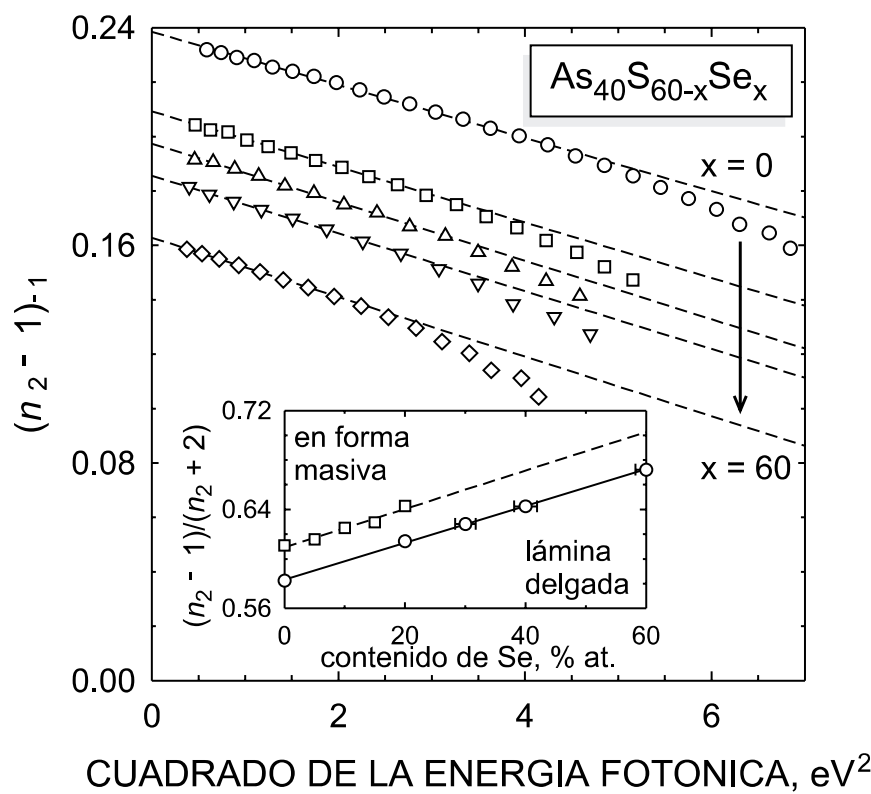

Figura 2. Representación de los valores del índice de refracción de las láminas del sistema $\mathrm{As}_{40} \mathrm{~S}_{60-\mathrm{x}} \mathrm{Se}_{x^{\prime}}$ en la forma $\left(n^{2}-1\right)^{-1}$ frente a $(\eta \omega)^{2}$, atendiendo a la Ec. (1). Las líneas discontinuas representan los correspondientes ajustes por mínimos cuadrados. La dependencia de los datos se aleja de la linealidad a medida que la absorción óptica se hace patente. En el recuadro se ha representado el factor del índice de refracción $\left(n^{2}-1\right) /\left(n^{2}+2\right)$ en función del contenido de Se, de acuerdo con la relación de Lorentz-Lorenz, para láminas amorfas (tomando los valores de $n(0)$ ) y para muestras masivas (tomando los valores de $n(\lambda=5 \mu \mathrm{m}))$ [6], dentro de la línea de composiciones $\mathrm{As}_{40} \mathrm{~S}_{60-\mathrm{x}} \mathrm{Se}_{\mathrm{x}}$.

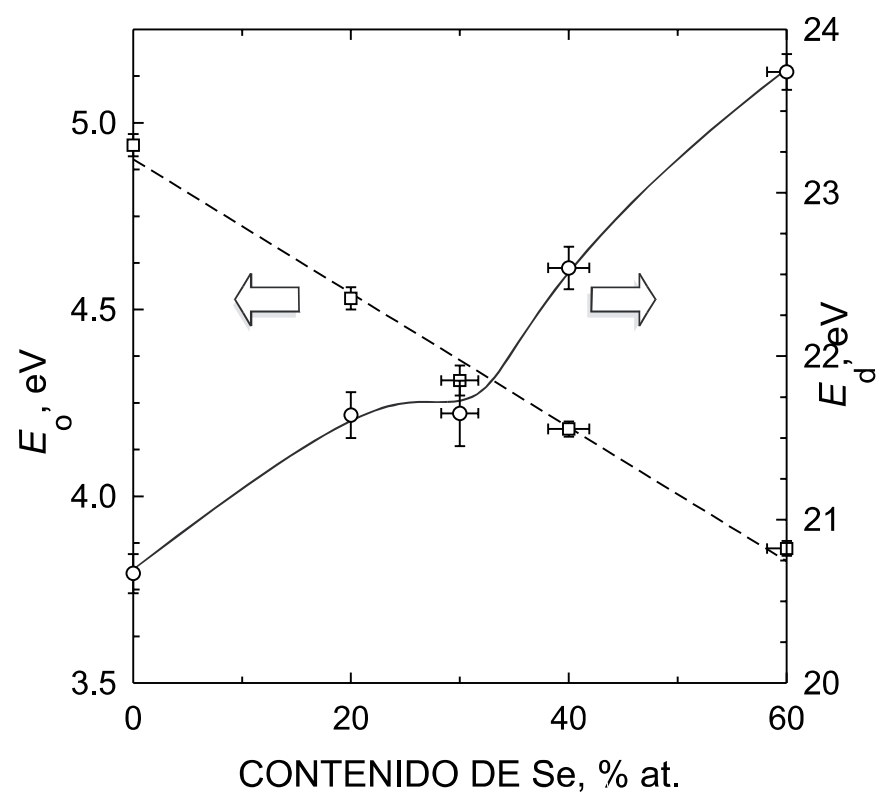

Figura 3. Parámetros de dispersión óptica, $E_{\mathrm{o}}$ y $E_{\mathrm{d}^{\prime}}$ frente al contenido de Se. Las líneas se han trazado como guías de la dependencia composicional que para estas magnitudes se infiere a partir de los resultados experimentales. 


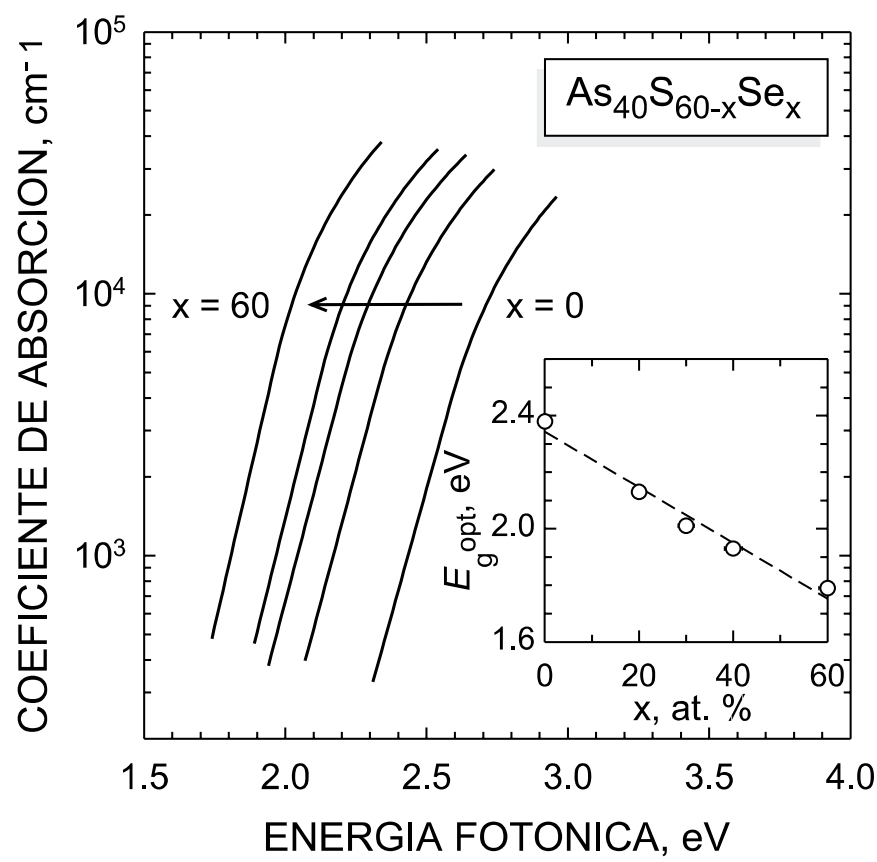

Figura 4. Espectros de absorción óptica, $\alpha(\eta \omega)$, para las láminas amorfas de composición $\mathrm{As}_{40} \mathrm{~S}_{60-x} \mathrm{Se}_{x}$. La dependencia composicional de $\mathrm{E}_{\mathrm{g}}^{\mathrm{opt}}$ se muestra en el recuadro. La línea se ha trazado como guía de la dependencia composicional que se infiere de los resultados experimentales.

ahora definimos formalmente. Los valores de $\mathrm{E}_{\mathrm{g}}^{\mathrm{opt}}$ correspondientes a las aleaciones $\mathrm{As}_{40} \mathrm{~S}_{60-\mathrm{x}} \mathrm{Se}_{\mathrm{x}}$ se han obtenido de la representación gráfica de $(\alpha \eta \omega)^{1 / 2}$ frente a $\eta \omega$, y dichos valores se han listado en la Tabla 2.

\section{DISCUSIÓN}

Como puede observarse en la Fig. 2, se ha encontrado un aumento en los valores del índice de refracción con el contenido de Se, dentro del intervalo espectral en estudio. Este aumento está relacionado con la mayor polarizabilidad, $\alpha_{\mathrm{p}^{\prime}}$ de los átomos de Se, más voluminosos (radio atómico, $115 \mathrm{pm}$ ), en comparación con los átomos de $\mathrm{S}$ (radio atómico, 100 pm). La polarizabilidad y el índice de refracción están conectados entre sí a través de la relación de Lorentz-Lorenz [19]:

$$
\frac{n^{2}-1}{n^{2}+2}=\frac{1}{3 \varepsilon_{0}} \sum_{\mathrm{j}} N_{\mathrm{j}} \alpha_{\mathrm{p}, \mathrm{j}}
$$

donde $\varepsilon_{0}$ es la permitividad en el vacío y $N_{\mathrm{j}}$ es el número de unidades polarizables del tipo $j$ por unidad de volumen, con polarizabilidad $\alpha_{p, j}$. La polarizabilidad $\alpha_{\mathrm{p}}$ que aparece en la Ec. (4), y que determina el índice de refracción, puede tener varios orígenes desde el punto de vista físico. En concreto, para sólidos predominantemente covalentes, en el intervalo óptico del espectro electromagnético, la polarizabilidad electrónica, la cual está asociada con la distorsión de la distribución electrónica de carga en un átomo, en relación con su ión correspondiente, así como con la distorsión de la densidad de carga electrónica en los enlaces covalentes, es dominante.

La Ec. (4) puede ser extendida a las diferentes contribuciones correspondientes a los elementos constituyentes de las aleaciones estudiadas, en la forma:

$$
\frac{n^{2}-1}{n^{2}+2}=\frac{1}{3 \varepsilon_{0}}\left[N_{\mathrm{As}} \alpha_{\mathrm{p}, \mathrm{As}}+N_{\mathrm{S}} \alpha_{\mathrm{p}, \mathrm{S}}+N_{\mathrm{Se}} \alpha_{\mathrm{p}, \mathrm{Se}}\right]
$$

Teniendo en cuenta la estequiometría particular de la línea composicional $\mathrm{As}_{40} \mathrm{~S}_{60-\mathrm{x}} \mathrm{Se}_{x^{\prime}}$ la Ec. (5) se puede rescribir como sigue:

$$
\frac{n^{2}-1}{n^{2}+2}=\frac{1}{3 \varepsilon_{0}}\left[\left(\frac{2}{3} \alpha_{\mathrm{p}, \mathrm{As}}+\alpha_{\mathrm{p}, \mathrm{S}}\right) N+\left(\alpha_{\mathrm{p}, \mathrm{Se}}-\alpha_{\mathrm{p}, \mathrm{S}}\right) N_{\mathrm{Se}}\right]
$$

donde $N=N_{\mathrm{S}}+N_{\mathrm{Se}}$ es una constante y $N_{\mathrm{As}}=2 / 3 N$. De acuerdo con la Ec. (6), cabe esperar una dependencia lineal entre el factor $\left(n^{2}-1\right) /\left(n^{2}\right.$ $+2)$ y $N_{\mathrm{Se}}$ (o de forma equivalente, $\mathrm{x}$ ). Las representaciones de este factor en función del contenido de Se, para nuestros valores de $n(0)$ y para los valores del índice de refracción a la longitud de onda $\lambda=5$ $\mu \mathrm{m}$ señalada por Sanghera et al. [6], para materiales en forma masiva con composiciones $\mathrm{As}_{40} \mathrm{~S}_{60-\mathrm{x}} \mathrm{Se}_{\mathrm{x}}(\mathrm{x}=0,5,10,15$ y $20 \%$ at.), se muestran en el recuadro de la Fig. 2. En ambos casos, los datos experimentales parecen estar en muy buen acuerdo con el comportamiento lineal predicho por la Ec. (6). Las diferencias existentes entre las dos representaciones recogidas en la Fig. 2 pueden explicarse basándose en la Ec. (4). Teniendo en cuenta que las muestras masivas tienen una densidad de masa más elevada que las láminas delgadas de la misma composición [1,2], la concentración de unidades polarizables, $N_{j^{\prime}}$ sería mayor para las muestras masivas que para las láminas recién evaporadas, lo que daría lugar a un también mayor valor del índice de refracción. Por otro lado, el paralelismo que existe entre ambas representaciones sugiere que las diferencias estructurales entre las muestras en forma masiva y las láminas recién evaporadas, las cuales afectarían a la contribución de $\alpha_{\mathrm{p}}$ asociada a los enlaces covalentes, no parecen ser muy relevantes en el caso de los valores del índice de refracción a las longitudes de onda consideradas, $n(0)$ y $n(\lambda=5 \mu \mathrm{m})$. De este modo, parece razonable pensar que la contribución atómica es el factor dominante para estos valores del índice de refracción.

En la Fig. 3 puede verse que la energía de dispersión, $E_{\mathrm{d}^{\prime}}$ aumenta al aumentar el contenido de Se. Teniendo en cuenta la Ec. (2), y asumiendo que los parámetros $N_{\mathrm{e}}=(40 \times 5+60 \times 6) / 60=28 / 3$ y $Z_{\mathrm{a}}=2$, mantienen los mismos valores a lo largo de la línea composicional en estudio, parece razonable adscribir la tendencia observada en los valores de $E_{\mathrm{d}}$ a un aumento en el número de coordinación efectivo del catión, $N_{c}$. Consideramos necesario mencionar, no obstante, la posible influencia del parámetro $\beta$ en el aumento observado en la energía de dispersión del oscilador. Así, la naturaleza del enlace químico cambiaría hacia menos iónico al aumentar el contenido de Se. Sin embargo, de acuerdo con la escala de electronegatividades de Pauling, un enlace As-S tiene un porcentaje de carácter iónico de $\approx 8 \%$ mientras que un enlace As-Se tiene un porcentaje de carácter iónico de $\approx 4 \%$. Por lo tanto, continuaremos nuestra discusión siguiendo el modelo original de WDD, en el que $\beta$ se considera una constante, con un valor covalente $\beta_{\mathrm{c}}=0.37 \pm 0.04 \mathrm{eV}$.

Es bien conocido [1] que la estructura de las aleaciones binarias calcogenuras del tipo $\mathrm{As}_{2} \mathrm{Ch}_{3}$ (siendo $\mathrm{Ch}$ un átomo calcogenuro), está constituida por capas estructurales bidimensionales formadas por unidades piramidales $\mathrm{AsCh}_{3^{\prime}}$ unidas a través de un átomo calcógeno bicoordinado, las cuales interaccionan entre sí mediante enlaces intermoleculares débiles. Además, se ha demostrado $[4,5,9]$ que esta estructura es también válida para los calcogenuros amorfos ternarios del sistema $\mathrm{As}_{40} \mathrm{~S}_{60-x} \mathrm{Se}_{x}$. En estos casos, se ha señalado $[4,5,9]$ la existencia de unidades piramidales mixtas del tipo $\operatorname{AsS}_{3-n} \mathrm{Se}_{\mathrm{n}}(\mathrm{n}=1$ ó 2$)$ en la matriz del material calcogenuro. De acuerdo con Wemple [16], la existencia de interacciones entre las capas estructurales a través de los átomos de As que actúan como puntos de enlace, formando enlaces intermoleculares As...Ch, contribuirían a un aumento en el número de coordinación efectivo del As, y por tanto cabría esperar un valor de $N_{\mathrm{c}}>3$. En particular, para la aleación amorfa binaria $\mathrm{As}_{40} \mathrm{~S}_{60}$ en forma masiva, Wemple sugiere un valor de $N_{c}=3.2$. Los valores de $N_{\mathrm{c}}$ para las láminas amorfas recién evaporadas de las aleaciones 
$\mathrm{As}_{40} \mathrm{~S}_{60-x} \mathrm{Se}_{x^{\prime}}$ obtenidos a partir de la Ec. (2), aparecen recogidos en la Tabla 2.

Siguiendo las ideas de Wemple [16], el aumento global observado en $E_{\mathrm{d}}$ (y como consecuencia, en $N_{\mathrm{c}}$ ) al aumentar el contenido de Se, apunta hacia una mayor interacción entre las capas estructurales, lo que es consistente con el aumento en la compactación estructural para los calcogenuros en forma masiva del sistema $\mathrm{As}_{40} \mathrm{~S}_{60-x} \mathrm{Se}_{x}$ referida por Sanghera et al. [6]. Es importante mencionar también, que recientemente Georgiev et al. [20] han señalado que en las aleaciones calcogenuras ricas en Se existen unidades cuasi-tetraédricas del tipo $\mathrm{Se}=\mathrm{As}\left(\mathrm{Se}_{1 / 2}\right)_{3^{\prime}}$ en las que se encuentran átomos de As tetracoordinados, además de los átomos de As tricoordinados correspondientes a las unidades piramidales $\mathrm{AsSe}_{3}$ Esta observación está también de acuerdo con que $N_{c}>3$, lo que apoya nuestros resultados. Por tanto, incluso aunque el aumento inferido para $N_{\mathrm{c}}$ en este trabajo está prácticamente dentro de las cotas de error (ver Tabla 2), pensamos que el aumento en $E_{\mathrm{d}^{\prime}}$ en el que se basa la conclusión anterior, es sin duda alguna significativo.

Por otro lado, Feltz [5] y Stronski et al. [21] han observado pequeñas desviaciones del volumen molar promedio, $\Delta V$, respecto a la suma de los volúmenes molares de los componentes, en los materiales en forma masiva del sistema $\left(\mathrm{As}_{2} \mathrm{~S}_{3}\right)_{1-\mathrm{y}}\left(\mathrm{As}_{2} \mathrm{Se}_{3}\right)_{\mathrm{y}^{\prime}}$ Estas desviaciones se han asociado con un alejamiento en la sustitución de $\mathrm{S}$ por Se, respecto al caso estadístico. Además, diferentes autores [1-3] han observado la presencia de fragmentos moleculares del tipo $\mathrm{As}_{4} \mathrm{~S}(\mathrm{Se})_{4^{\prime}} \mathrm{S}(\mathrm{Se})_{\mathrm{n}}$ y $\mathrm{As}_{4}$ en la matriz de láminas recién depositadas de aleaciones tanto binarias como ternarias del sistema $\mathrm{As}_{40} \mathrm{~S}_{60-\mathrm{x}} \mathrm{Se}_{\mathrm{x}^{\prime}}$ preparadas por evaporación térmica en vacío. Estos fragmentos moleculares dificultarían la cohesión entre las capas estructurales y consecuentemente, contribuirían también a un aumento de $\Delta V$, así como a una disminución del número de coordinación efectivo, $N_{\text {c }}$. Esto explicaría la diferencia entre el valor de $N_{\mathrm{c}}$ encontrado para nuestras láminas amorfas de composición $\mathrm{As}_{40} \mathrm{~S}_{60^{\circ}} N_{\mathrm{c}} \approx 3.0$, y el valor inferido por Wemple [16] para los materiales en forma masiva de la misma composición, $N_{\mathrm{c}}=3.2$. Así, las desviaciones con respecto a la sustitución estadística de $\mathrm{S}$ por Se, así como la presencia de los fragmentos moleculares anteriormente mencionados, podrían estar relacionadas con la dependencia no lineal observada en la energía de dispersión, $E_{d}$ (ver Fig. 3).

Las anteriores ideas sugeridas para explicar el comportamiento encontrado para $E_{\mathrm{d}^{\prime}}$ se ven reforzadas, además, por los resultados obtenidos mediante difracción de rayos X, los cuales se muestran en la Fig. 5. En esta figura, aparte de la ausencia de picos cristalinos, que pone de manifiesto el carácter amorfo de estas láminas, se observa en todos los casos, con mayor o menor intensidad, el denominado pre-pico de difracción o first sharp diffraction peak (FSDP), en su terminología inglesa. Esta característica de los patrones de difracción de los sólidos amorfos se ha relacionado con la presencia de zonas de baja ocupación atómica, o intersticios, alrededor de las unidades estructurales que conforman la matriz amorfa [22,23]. Resulta claro que los fragmentos moleculares mencionados anteriormente, que están presentes en las láminas recién evaporadas, favorecerían la presencia de estos intersticios y, a su vez, se reflejarían en la intensidad del FSDP. Así, en los patrones de difracción presentados en la Fig. 5 se observa una disminución gradual en la intensidad del FSDP con el contenido de Se. Este decrecimiento no es proporcional a $\mathrm{x}$, y parece haber un mínimo en la intensidad del FSDP para alguna composición intermedia en el sistema $\mathrm{As}_{40} \mathrm{~S}_{60-x} \mathrm{Se}_{x}$. Este resultado pensamos que podría estar relacionado con la dependencia no lineal observada en los valores de $E_{\mathrm{d}}$. Queremos resaltar que este tipo de dependencia no lineal ha sido encontrada también por Vlcek et al. [9] en otras magnitudes físicas, como son la temperatura de transición vítrea, $T_{\mathrm{g}^{\prime}}$ la entalpía de relajación, $\Delta H$, y la capacidad calorífica específica, $C_{\mathrm{p}^{\prime}}$ en el caso de materiales amorfos en forma masiva del mismo

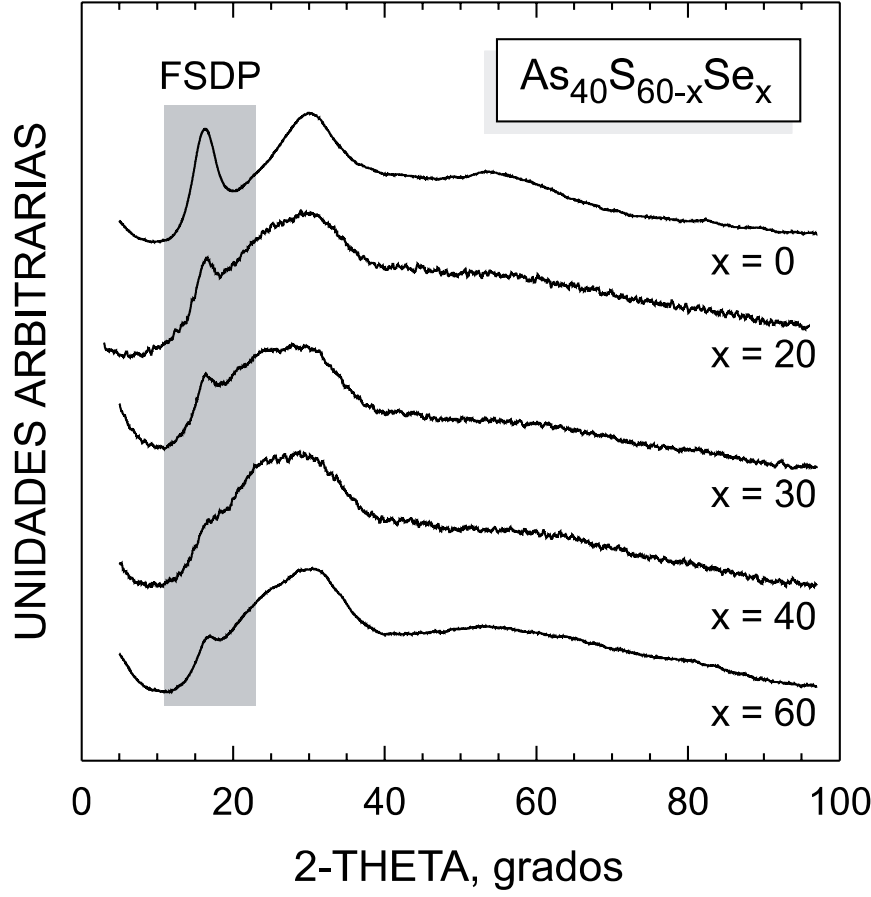

Figura 5. Difractogramas de rayos $X$ de las láminas amorfas del sistema $\mathrm{As}_{40} \mathrm{~S}_{60-x} \mathrm{Se}_{\mathrm{x}}$. Se ha sombreado la región en la que aparece el FSDP, para destacar esta importante característica relacionada con el orden de medio alcance de los sólidos amorfos.

sistema $\mathrm{As}_{40} \mathrm{~S}_{60-x} \mathrm{Se}_{x^{\prime}}$ así como en el parámetro de dispersión $E_{\mathrm{d}^{\prime}}$ en el caso de láminas recocidas de este sistema concreto.

Finalmente, se ha encontrado una disminución lineal en el valor de $\mathrm{E}_{\mathrm{g}}^{\text {opt }}$ con el aumento en el contenido de $\mathrm{Se}$, como se observa en el recuadro de la Fig. 4. Una dependencia composicional similar a ésta ha sido observada para la energía del oscilador, $E_{\mathrm{o}}$ (ver Fig. 3), como era de esperar de acuerdo con la ecuación presentada anteriormente, $E_{\mathrm{o}} \approx 2 \times \mathrm{E}_{\mathrm{g}}^{\mathrm{opt}}$ [17]. La disminución de ambos parámetros ópticos puede explicarse en base a la mayor energía de los enlaces As-S (379.5 $\left.\mathrm{kJ} \mathrm{mol}^{-1}\right)$, en comparación con la energía de los enlaces As-Se $\left(96 \mathrm{~kJ} \mathrm{~mol}^{-1}\right)$. Es importante resaltar que las especies moleculares que existen en las láminas amorfas recién evaporadas del sistema $\mathrm{As}_{40} \mathrm{~S}_{60-\mathrm{x}} \mathrm{Se}_{\mathrm{x}}\left(\mathrm{As}_{4} \mathrm{~S}(\mathrm{Se})_{4^{\prime}} \mathrm{S}(\mathrm{Se})\right.$ y $\left.\mathrm{As}_{4}\right)$, introducen enlaces homopolares de los tipos As-As, S-S y Se-Se. Así, incluso aunque las más altas energías de tales enlaces homopolares (382.0, 425.3 y $332.6 \mathrm{~kJ} \mathrm{~mol}^{-1}$, respectivamente) podrían de algún modo contribuir a un aumento en el valor de $\mathrm{E}_{\mathrm{g}}^{\mathrm{opt}}$ y $E_{\mathrm{o}^{\prime}}$ de acuerdo con nuestros resultados, no parece que tengan un papel importante en el comportamiento de ambos parámetros al cambiar $\mathrm{x}$.

\section{CONCLUSIONES}

Las propiedades ópticas de láminas amorfas recién depositadas de composiciones $\mathrm{As}_{40} \mathrm{~S}_{60-\mathrm{x}} \mathrm{Se}_{\mathrm{x}}$ (con $\mathrm{x}=0$, 20, 30, 40 y $60 \%$ at.), preparadas mediante evaporación térmica, se han calculado a partir de los correspondientes espectros de transmisión óptica, obtenidos en incidencia normal. El espesor medio, , y el índice de refracción, $n$, de las láminas, se han determinado con una precisión mejor que el $1 \%$. Para ello se ha empleado un método de caracterización óptica basado en las envolventes de los espectros de transmisión, y se ha tenido en cuenta la absorción del substrato vítreo sobre el que las láminas están depositadas. Hemos encontrado que el índice de refracción aumenta con el contenido de Se sobre todo el rango espectral objeto de estudio, 
400-2200 nm. El análisis de la dependencia composicional del índice de refracción, sobre la base de la relación de Lorentz-Lorenz, sugiere que este aumento está relacionado con la mayor polarizabilidad, $\alpha_{\mathrm{p}^{\prime}}$ de los átomos de Se, en comparación con los átomos de S. El parámetro de dispersión, $E_{\mathrm{d}^{\prime}}$ crece de forma no lineal con el aumento de $\mathrm{x}$, presentando una meseta alrededor de $\mathrm{x} \approx 30 \%$ at. De acuerdo con el modelo sugerido por Wemple [16], se ha inferido que el aumento de $E_{\mathrm{d}}$ apunta hacia una mayor interacción entre las capas estructurales, dando lugar de este modo a un aumento en el número de coordinación efectivo de los átomos de As, $N_{c}$. No obstante, tampoco puede excluirse una disminución en el carácter iónico del enlace químico con el aumento en el contenido de Se, y su posible influencia en el valor del parámetro $\beta$. Por otra parte, el comportamiento no lineal pensamos que podría estar relacionado con pequeñas desviaciones en la sustitución de $\mathrm{S}$ por Se, respecto al caso estadístico, así como la presencia de fragmentos moleculares en el seno de la matriz amorfa de las láminas recién evaporadas. Los resultados obtenidos mediante difracción de rayos $\mathrm{X}$ parecen apoyar esta conclusión. Por último, se ha observado una disminución lineal en los parámetros $E_{\mathrm{o}} \mathrm{y} \mathrm{E}_{\mathrm{g}}^{\mathrm{opt}}$, lo cual puede explicarse teniendo en cuenta la mayor energía de los enlaces As-S, en comparación con la correspondiente a los enlaces As-Se.

\section{AGRADECIMIENTOS}

Este trabajo ha sido parcialmente financiado por el MCYT y FEDER, como parte del Proyecto MAT2001-3333, y a través de una beca Marie Curie del Programa de la Comunidad Europea "Improving Human Research Potential and the Socio-Economic Knowledge Base", bajo el contrato HPMF-CT-2000-01031.

\section{BIBLIOGRAFÍA}

1. S.R. Elliott, "Chalcogenide glasses", en Materials Science and Technology, Cap. 7, Vol. 9, J. Zarzycki, ed. (VCH, Weinheim, 1991), p. 375.

2. M.A. Popescu, Non-Crystalline Chalcogenides (Kluwer Academic Publishers, Dordrecht, 2000).

3. A.V. Kolobov, Ka. Tanaka, "Photoinduced phenomena in amorphous chalcogenides: from phenomenology to nanoscale", en Handbook of Advanced Electronic and Photonic Materials and Devices, Vol. 5, Cap. 2, Hari Singh
Nalwa, ed. (Academic Press, San Diego, 2001), p. 47.

4. J.A. Freitas, U. Strom, D.J. Treacy, "Raman-scattering of the mixed chalcogenide glass system $\mathrm{As}_{2} \mathrm{~S}_{x} \mathrm{Se}_{3-x}$ ", J. Non-Cryst. Solids 59\&60 (1983) 875.

5. A. Feltz, "Amorphous and inorganic materials and glasses" (VCH, Weinheim, 1993).

6. J.S. Sanghera, V.W. Nguyen, I.D. Aggarwal, “Properties of $\mathrm{As}_{40} \mathrm{~S}_{60-\mathrm{x}} \mathrm{Se}_{\mathrm{x}}$ glasses for IR fiber optics", J. Am. Ceram. Soc. 79 (1996) 1324.

7. E. Márquez, J.M. González-Leal, R. Prieto-Alcón, M. Vlcek, A. Stronski, T. Wagner, D. Minkov, “Optical characterization of thermally evaporated thin films of $\mathrm{As}_{40} \mathrm{~S}_{40} \mathrm{Se}_{20}$ chalcogenide glass by reflectance measurements", Appl. Phys. A 67 (1998) 371.

8. E. Márquez, J.M. González-Leal, R. Prieto-Alcón, R. Jiménez-Garay, M. Vlcek, "On the photo- and thermally-induced darkening phenomena in as $_{40} \mathrm{~s}_{40} \mathrm{se}_{20}$ amorphous chalcogenide thin films", J. Phys. D: Appl. Phys. 32 (1999) 3128.

9. M. Vlcek, A.V. Stronski, A. Sklenar, T. Wagner, S.O. Kasap, "Structure and imaging properties of $\mathrm{As}_{40} \mathrm{~S}_{60-\mathrm{x}} \mathrm{Se}_{\mathrm{x}}$ glasses", J. Non-Cryst. Solids 266-269 (2000) 964.

10. A.V. Stronski, M. Vlcek, A. Sklenar, P.E. Shepeljavi, S.A. Kostyukevich, T. Wagner, "Application of $\mathrm{As}_{40} \mathrm{~S}_{60-\mathrm{x}} \mathrm{Se}_{\mathrm{x}}$ layers for high-efficiency grating production", J. Non-Cryst. Solids 266-269 (2000) 973.

11. E. Márquez, J.M. González-Leal, R. Jiménez-Garay, M. Vlcek, “Thermallyand photo-induced changes in the structure and optical properties of amorphous $\mathrm{As}_{40} \mathrm{~S}_{30} \mathrm{Se}_{30}$ films", Thin Solid Films 396 (2001) 183.

12. J.S. Berkes, S.W. Ing. Jr., W.J. Hillegas, J. Appl. Phys. 42 (1971) 4908.

13. J.M. González-Leal, R. Prieto-Alcón, J.A. Angel, D.A. Minkov, E. Márquez, "Influence of the substrate absorption on the optical and geometrical characterisation of thin dielectric films", Appl. 41 (2002) 7300.

14. M. McClain, A. Feldman, D. Kahaner, X. Ying, "An algorithm and computer program for the calculation of envelope curves", Comput. Phys. 5 (1991) 45.

15. S.H. Wemple, M. DiDomenico, "Behavior of the electronic dielectric constants in covalent and ionic materials", Phys. Rev. B 3 (1971) 1338.

16. S.H. Wemple, "Refractive-Index behavior of amorphous semiconductors and glasses", Phys. Rev. B 7 (1973) 3767.

17. Ke. Tanaka, "Optical properties and photoinduced changes in As-S films" Thin Solid Films 66 (1980) 271.

18. J. Tauc, A. Menth, "States in the gap", J. Non-Cryst. Solids 8-10 (1972) 569.

19. S.R. Elliott, "The physics and chemistry of solids" (Wiley, Chichester, 2000).

20. D.G. Georgiev, P. Boolchand, M. Micoulaut, "Rigidity transitions and molecular structure of As $\mathrm{Se}_{1-\mathrm{x}}$ glasses", Phys. Rev. B 62 (2000) R9228.

21. A.V. Stronski, M. Vlcek, P.F. Oleksenko, "Fourier raman spectroscopy studies of the $\mathrm{As}_{40} \mathrm{~S}_{60-x} \mathrm{Se}_{x}$ glasses", Semiconductors Physics, Quantum Electronics \& Optoelectronics 4 (2001) 210.

22. S.R. Elliott, "Medium-range order in the structure of amorphous solids", Nature 354 (1991) 445.

23. S.R. Elliott, "The origin of the first sharp diffraction peak in the structure of covalent glasses", Phys. Rev. Lett. 67 (1991) 711.

Recibido: 1.2 .03

Aceptado: 30.11 .03 